\title{
3D Model Retrieval Using 3D-DFT Spectral and Cepstral Descriptors of Local Octants
}

\author{
Chang-Hsing Lee*, Jau-Ling Shih, and Yu-Hau Liu \\ Department of Computer Science and Information Engineering \\ Chung Hua University, Hsinchu, Taiwan \\ *Corresponding Author: chlee@ chu.edu.tw
}

\begin{abstract}
Conventional 3D-DFT features proposed for 3D model retrieval tried to extract the spectral/cepstral features from the spectrum/cepstrum of the 3D model, without considering the local properties of a 3D model. In this paper, local 3D-DFT spectral descriptor as well as local 3D-DFT cepstral descriptor will be proposed for 3D model retrieval. First, the set of voxels covering the surface of a 3D model is divided into 8 octants. 3D-DFT spectral descriptors and 3D-DFT cepstral descriptors are extracted from each octant and combined to form the local 3D-DFT spectral descriptor and local 3D-DFT cepstral descriptor. Experiments conducted on the Princeton Shape Benchmark (PSB) database have shown that the proposed local 3D-DFT descriptors outperform other 3D-DFT descriptors.
\end{abstract}

Keywords: 3D model retrieval, 3D discrete Fourier transform (3D-DFT), 3D-DFT spectrum, 3D-DFT cepstrum

\section{Introduction}

3D models have been widely used in computer aided design, computer animations, movie production, industrial product design, digital library, and so on. Since the number of 3D models grows rapidly, there exist increasing demands for 3D model retrieval systems. Typically, keyword-based search systems were provided to find similar images, videos, or 3D models. To facilitate 3D model retrieval using keyword search mechanism, each 3D model must be labeled with some appropriate keywords. However, it is time-consuming to manually annotate each 3D model with appropriate keywords. Further, it is sometimes hard to find appropriate keywords for some specific models. To overcome this problem, content-based 3D model retrieval mechanism has become widely used to find similar 3D models.

The main challenge to a content-based 3D model retrieval system is how to extract representative features for effectively discriminating different types of 3D models [1, 2]. In general, 3D model retrieval methods can be classified into four categories: histogram-based descriptors [3-19], transform-based descriptors [20-28], 2D view-based descriptors [29-52], and graph-based descriptors [53-56].

Histogram-based descriptors consider the statistical distributions or histograms of local features evaluated at the vertices or meshes of a 3D model. These histogram-based descriptors include geodesic features [3,4], local statistical features [5], Poisson histogram [6], sliced image histogram [7], beta/distance histograms (BD) [8], grid D2 (GD2) [9], modified D2 descriptor [10, 11], shape distributions [13], shape spectrum descriptor (SSD) [14, 15], 3D Hough transform descriptors [16], shape histograms [19], etc. The main drawback of these spatial shape descriptors is that they do not take into account how the local features are spatially distributed over the model surface.

Transform-based descriptors are extracted by first mapping the 3D data into frequency domain. These frequency domain representations include probability density-based shape descriptors [21], concrete radicalized spherical [22], spherical wavelet transform [23], 3D discrete curvelet transform [24], rotation invariant spherical harmonics [1, 25], spherical harmonics [26], 3D Fourier transform [27], angular radial transform (ART) [28], etc. The effectiveness of these frequency descriptors relies heavily on the resolution of the voxelization (voxel decomposition) operation of a 3D model and how to extract discriminative features from the set of transformed coefficients. 
2D view-based descriptors are generally obtained by projecting a 3D model on a number of $2 \mathrm{D}$ planes from different views. These projected results can be either binary images representing the silhouettes from different views, or gray-level images representing the curvature, depth, or some geometric information. One merit of the view-based descriptors is that it is easy to design a query interface which supports a $2 \mathrm{D}$ sketch for $3 \mathrm{D}$ model retrieval. The view-based descriptors include camera constraint free view-based descriptor [29], Hypergraph [30], Bayesian network lightfield descriptor (BLD) [32], weighted bipartite graph matching (WBGM) [32], PCA plane projection [33], spatial shape information [34], Bag-of-Features [35], spatial structure circular descriptor (SSCD) [36], Panoramic views [37], shape impact descriptor (SID) [38], ART elevation descriptor (AED) [39], Multi-Fourier spectra descriptor [40], principal plane analysis [41, 42], spherical trace transform [43], depth image [44], elevation descriptor (ED) [45], curvature map [46-48], grid sphere descriptor (GSD) [49], depth buffer descriptor [50], light field Descriptor (LFD) [51], etc. The major problem with view-based descriptors is that rotation invariance has to be solved by either pose normalization prior to $2 \mathrm{D}$ projections, by extracting rotation-invariant features, or by matching $2 \mathrm{D}$ feature descriptors over many different alignments.

Graph-based descriptors have the potential of describing the geometrical and topological properties of a 3D model in a more faithful way, particularly for deformable 3D models. The methods include extended cone-curvature [53], medial scaffold [54], Reeb graph [55], skeleton-based descriptors [56], etc.

In our prior work [57], we have designed a 3D model retrieval system using 3D discrete Fourier transform (3D-DFT) spectral features and cepstral features. Experimental results have shown that these descriptors can obtain promising performances. In this paper, local 3D-DFT spectral and cepstral features will be extracted from different local regions of the $3 \mathrm{D}$ model and combined for 3D model retrieval.

\section{Review of 3D-DFT Descriptors}

In this section, we will first describe some 3D-DFT feature extraction methods for 3D model retrieval, including 3D-DFT spectrum [27, 57], 3D-DFT cepstrum [57], 3D-DFT subband spectrum [57], and 3D-DFT subband cepstrum [57].

\section{$2.1 \quad 3 D-D F T$ Spectrum}

The 3D-DFT spectrum was obtained by applying 3D-DFT on the voxels of a 3D model $V(x, y, z)$ defined as follows [27]:

$$
F(u, v, w)=\frac{1}{\sqrt{N^{3}}} \sum_{x=0}^{N-1} \sum_{y=0}^{N-1} \sum_{z=0}^{N-1} V(x, y, z) \exp \left(\frac{-j 2 \pi(u x+v y+w z)}{N}\right)
$$

where $u, v$ and $w$ are 3D-DFT frequency indices $(0 \leq u, v, w$ $<N)$. The magnitudes of those low-frequency coefficients (except $|F(0,0,0)|$ ) were selected to form the 3D-DFT spectral features.

\subsection{D-DFT Cepstrum}

By applying 3D-IDFT on the magnitude spectrum $\mid F(u$, $v, w)$, we can obtain the 3D-DFT cepstrum $C(a, b, c)$ :

$$
C(a, b, c)=\frac{1}{\sqrt{N^{3}}} \sum_{u=0}^{N-1} \sum_{v=0}^{N-1} \sum_{w=0}^{N-1}|F(u, v, w)| \exp \left(\frac{j 2 \pi(a u+b v+c w)}{N}\right)
$$

where $a, b$ and $c$ are 3D-DFT quefrency (cepstral frequency) indices. The magnitudes of those low-quefrency coefficients were selected to form the 3D-DFT cepstral features.

\subsection{D-DFT Subband Spectrum}

To obtain the 3D-DFT subband spectral features, we first decompose the 3D-DFT spectrum into $K(K=W \times H \times L)$ subbands, notated by $S B_{p, q, r}(0 \leq p<W, 0 \leq q<H, 0 \leq r<$ $L$ ), by using uniform subband decomposition or logarithmic subband decomposition along the $x$-axis, $y$-axis, and $z$-axis, respectively [57]. Then, the sum of magnitude spectrum within each subband, notated by $M_{p, q, r}$, is calculated to yield the 3D-DFT subband spectral features:

$$
M_{p, q, r}=\sum_{(u, v, w) \in S B_{p, q, r}}|F(u, v, w)|
$$

\subsection{D-DFT Subband Cepstrum}

By applying 3D-IDFT on the subband magnitude spectrum, we can obtain the 3D-DFT subband cepstrum:

$$
C_{S B}(a, b, c)=\frac{1}{\sqrt{N^{3}}} \sum_{p=0}^{W-1} \sum_{q=0}^{H-1} \sum_{r=0}^{L-1} M_{p, q, r} \exp \left(\frac{j 2 \pi(a p+b q+c r)}{N}\right)
$$

The magnitudes of those low-quefrency coefficients (except $\left.\left|C_{S B}(0,0,0)\right|\right)$ were selected as the 3D-DFT subband cepstral features.

\section{Proposed 3D Model Retrieval Approach}

Prior 3D-DFT feature extraction methods tried to extract the spectral/cepstral features from the spectrum/cepstrum of the 3D model, without considering 
the local properties of a 3D model. Thus, in this paper, we will try to extract local spectral/cepstral features to capture the local characteristics of different parts of a 3D model. First, the principal planes method [42] is used for pose alignment of a 3D model. Then, the 3D model is divided into eight octants. 3D-DFT spectral/cepstral features [27, 57] will then be extracted from each local octant. Finally, the spectral and cepstral features extracted from all octants will be combined to search similar 3D models.

\subsection{D Model Alignment}

In this paper, we use the principal planes method [42] to align the pose of each 3D model. First, the smallest bounding box that circumscribes the $3 \mathrm{D}$ model is divided into a voxel grid of size $N \times N \times N$ (please see Fig. 1). In this paper, $N=64$. If there is a polygonal surface passes through the voxel with coordinates $(x, y, z)$, this voxel is defined as an opaque voxel, notated by $V(x, y, z)=1$; otherwise, this voxel is defined as a transparent voxel, notated by $V(x, y, z)$ $=0$. As a result, the voxelization operation converts the $3 \mathrm{D}$ mesh model into a discrete $3 \mathrm{D}$ function with regularly sampled voxels in 3D coordinates. Second, the model's mass center is moved to $(N / 2, N / 2, N / 2)$. The $3 \mathrm{D}$ model is then scaled such that the average distance from all opaque voxels to the mass center becomes N/4. Third, the pose of this $3 \mathrm{D}$ model is aligned by the grid-based principal component analysis (GPCA) [33]. Thus, the features extracted from this aligned 3D model will be invariant to rotation, scaling, and translation.

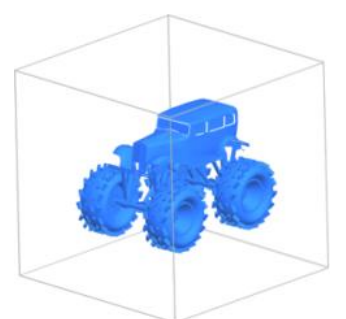

(a)

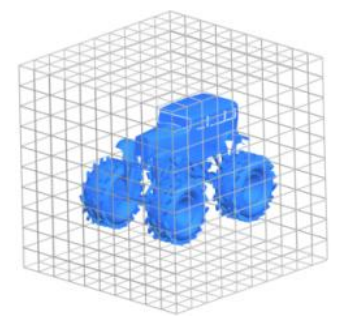

(b)

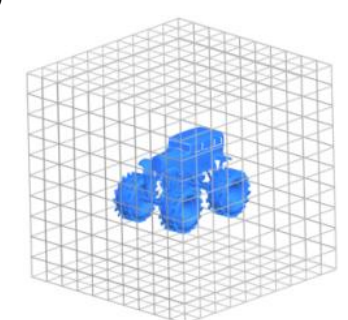

(c)
Fig. 1. 3D model alignment. (a) The 3D monster truck model circumscribed by a bounding box. (b) The bounding box is decomposed into a $N \times N \times N$ voxel grid. (c) The normalized 3D monster truck model.

\subsection{Inverse Distance Transform}

The voxelization operation will encode each voxel in the 3D Cartesian coordinate system a value 1 (denoting an opaque voxel) or 0 (denoting a transparent voxel). Such a binary encoding method will produce severe high-frequency artifacts due to the blocky structure of the binary voxel representation. To suppress these high-frequency artifacts, we will employ the inverse distance transform (IDT) proposed by Dutagaci et al. [20] to get a smoothed voxel representation. The IDT value associated with a voxel $V$ is defined as follows:

$$
I D T(V)=\frac{1}{1+D T(V)}
$$

where $D T(V)$ is the distance between $V$ and its nearest opaque voxel in the $3 \mathrm{D}$ space given by

$$
D T(V)=\min _{V_{o} \in \mathbf{V}^{0}} d\left(V, V_{O}\right)
$$

where $d\left(V, V_{O}\right)$ is the distance between voxel $V$ and an opaque voxel $V_{O}$ measured by the Euclidean distance, $\mathbf{V}^{\mathbf{o}}$ is the set of all opaque voxels:

$$
\mathbf{V}^{\mathbf{o}}=\{V \mid V(x, y, z)=1\}
$$

Therefore, the voxels on the surface of the 3D model take the largest IDT values and the IDT value decreases smoothly as the voxels move away from the model surface. Fig. 2 gives an example showing how the IDT operation was used to get a smoothed voxel representation.

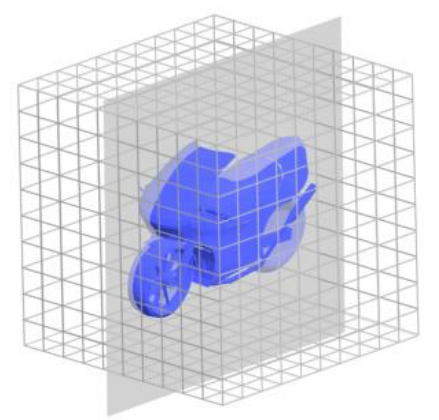

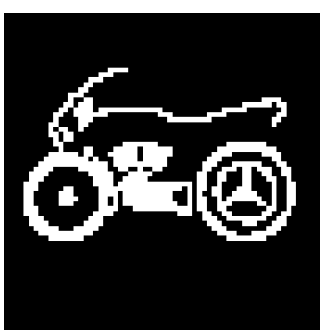

(b) (a)

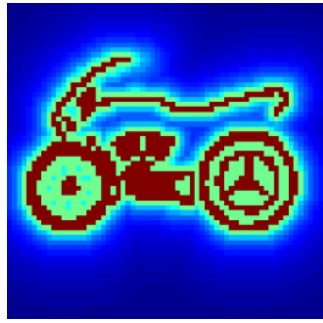

(c)
Fig. 2. An example illustrating IDT. (a) A slice of the motorcycle model (b) A slice from binary voxel respresentation (c) A slice resulted from IDT operation 


\subsection{D Model Decomposition}

To extract the local features, we divide the set of voxels of a $3 \mathrm{D}$ model in the 3D space into 8 octants represented as follows:

$$
\begin{aligned}
& Q_{1}=\{(x, y, z) \mid x \geq 0, y \geq 0, z \geq 0\} \\
& Q_{2}=\{(x, y, z) \mid x<0, y \geq 0, z \geq 0\} \\
& Q_{3}=\{(x, y, z) \mid x<0, y<0, z \geq 0\} \\
& Q_{4}=\{(x, y, z) \mid x \geq 0, y<0, z \geq 0\} \\
& Q_{5}=\{(x, y, z) \mid x \geq 0, y \geq 0, z<0\} \\
& Q_{6}=\{(x, y, z) \mid x<0, y \geq 0, z<0\} \\
& Q_{7}=\{(x, y, z) \mid x<0, y<0, z<0\} \\
& Q_{8}=\{(x, y, z) \mid x \geq 0, y<0, z<0\}
\end{aligned}
$$

where $Q_{i}(1 \leq i \leq 8)$ denote the $i$-th octant (see Fig. 3). Thus, each octant contains some specific voxels (revealing local properties) of a 3D model. Since the size of the bounding box is $N \times N \times N$, the size of each local octant is $N / 2 \times N / 2 \times N / 2$. For each local octant, the 3D-DFT spectral and 3D-DFT cepstral features will be extracted and concatenated to form the local 3D-DFT spectral descriptor and local 3D-DFT cepstral descriptor.

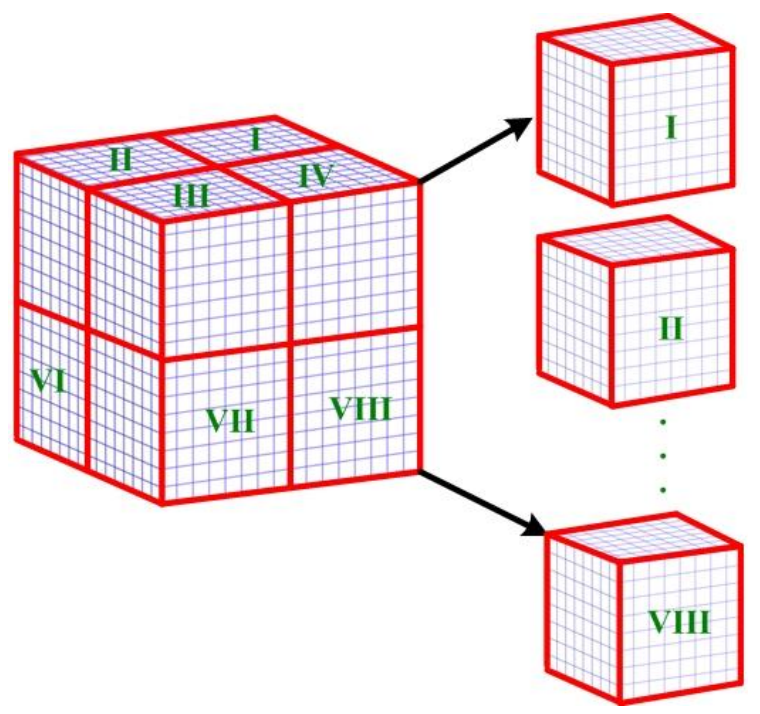

Fig. 3. Decomposition of a 3D model into 8 octants.

\subsection{Local 3D-DFT Spectrum}

The local 3D-DFT spectrum is obtained by applying $3 \mathrm{D}$-DFT on the voxels within each octant $Q_{i}(1 \leq i \leq 8)$ to get the corresponding local spectrum:

$$
F_{i}(u, v, w)=\frac{1}{\sqrt{N^{3} / 8}} \sum_{(x, y, z) \in Q_{i}} V(x, y, z) \exp \left(\frac{-j 2 \pi(u x+v y+w z)}{N / 2}\right)
$$

where $u, v$ and $w$ are 3D-DFT frequency indices $(0 \leq u, v, w$ $<N), F_{i}$ is the spectrum of the $i$-th octant. The magnitudes of those $M \times M \times M$ low-frequency coefficients (except $\mid F_{i}(0$, $0,0) \mid$ ) of each local spectrum $F_{i}$ will be concatenated to form the local 3D-DFT spectral descriptor. Thus, the length of the local 3D-DFT spectral descriptor is $8\left(M^{3}-1\right)$.

\subsection{Local 3D-DFT Cepstrum}

By applying 3D-IDFT on each local magnitude spectrum $\left|F_{i}(u, v, w)\right|$, we can obtain the local 3D-DFT cepstrum $C_{i}(a, b, c)$, where $a, b$ and $c$ are 3D-DFT quefrency indices. The magnitudes of those $D \times D \times D$ low-quefrency coefficients (except $\left.\left|C_{i}(0,0,0)\right|\right)$ of each local cepstrum $C_{i}$ will be concatenated to form the local 3D-DFT cepstral descriptor. Thus, the length of the local 3D-DFT cepstral descriptor is $8\left(D^{3}-1\right)$.

\subsection{D Model Retrieval}

Let $\mathbf{f}^{L S}$ denote the feature vector corresponding to the local 3D-DFT spectral descriptor of the query model, and $\mathbf{f}^{L S, t}$ denote the feature vector corresponding to the local 3D-DFT spectral descriptor of the $t$-th matching model in the database. The distance between the query model and the $t$-th matching model evaluated in terms of local 3D-DFT spectral descriptor is defined as follows:

$$
d^{L S, t}=\sum_{m=1}^{8\left(M^{3}-1\right)}\left|f^{L S}(m)-f^{L S, t}(m)\right|
$$

where $f^{L S}(m)$ and $f^{L S, t}(m)$ denote respectively the $m$-th feature value of $\mathbf{f}^{L S}$ and $\mathbf{f}^{L S, t}$.

Similarly, let $\mathbf{f}^{L C}$ and $\mathbf{f}^{L C, t}$ denote the local 3D-DFT cepstral descriptors of the query model and the $t$-th matching model, respectively. The distance between the query model and the $t$-th matching model evaluated in terms of local 3D-DFT cepstral descriptor is given as follows:

$$
d^{L C, t}=\sum_{d=1}^{8\left(D^{3}-1\right)}\left|f^{L C}(d)-f^{L C, t}(d)\right|
$$


where $f^{L C}(d)$ and $f^{L C, t}(d)$ denote respectively the $d$-th feature value of $\mathbf{f}^{L C}$ and $\mathbf{f}^{L C, t}$. Therefore, the models that are most similar to the query one can be found by finding those having smaller distance values.

\section{Experimental Results}

To demonstrate the effectiveness of the proposed local descriptor, some experiments have been conducted on the Princeton Shape Benchmark (PSB) database [58]. The PSB database contains 1814 models (161 classes) which are divided into 907 training models (90 classes) and 907 testing models (92 classes). The experiments were performed on the 907 testing models (see Fig. 3 and Fig. 4) to keep the results comparable with other methods. The discounted cumulative gain (DCG) [59] will be employed to compare the performance of different descriptors. DCG at the $r$-th rank is defined as follows:

$$
D C G_{r}= \begin{cases}D C G_{r-1}+\frac{L_{r}}{\log _{2}(r)} & r \geq 2 \\ L_{1} & r=1\end{cases}
$$

where $L_{r}=1$ if the query model and the $r$-th model in the ranked retrieval list have the same class label; otherwise, $L_{r}$ $=0$. The overall $D C G$ score for a query model is defined as $D C G_{T}$, where $T$ is the number of models in the database. It is clear that if the models appearing in the front of the retrieval list have the same class label as the query one, a larger $D C G$ score can be obtained. On the other hand, if the models having identical class label to the query one appear in the rear of the retrieval list, a small $D C G$ score will be obtained. In our experiments, each model in the database will be presented as a query one to evaluate the average $D C G$ score among all query models:

$$
D C G=\frac{1}{T} \sum_{t=1}^{T} D C G_{T}(t)
$$

where $D C G_{T}(t)$ denotes the $D C G$ score associated with the $t$-th matching model.

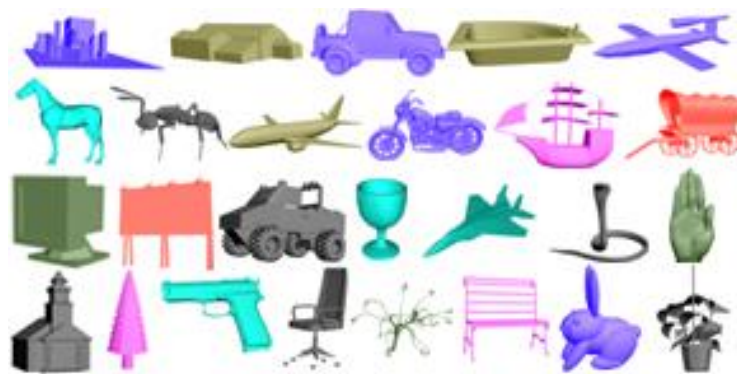

Fig. 3. Some 3D model classes in the PSB databse [58].

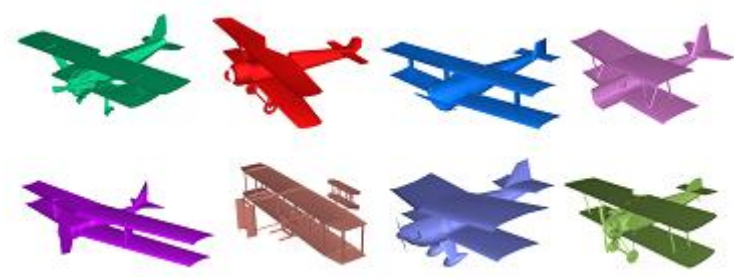

Fig. 4. The biplane class in the PSB databse [58].

Table 1 compared the performance of different 3D-DFT descriptors, including (1) 3D-DFT spectrum (notated by 3D-DFT-S), (2) 3D-DFT cepstrum (notated by 3D-DFT-C), (3) 3D-DFT subband spectrum with uniform subband decomposition (notated by 3D-DFT-S-USD), (4) 3D-DFT subband spectrum with logarithmic subband decomposition (notated by 3D-DFT-S-LSD), (5) 3D-DFT subband cepstrum with uniform subband decomposition (notated by 3D-DFT-C-USD), (6) 3D-DFT subband cepstrum with logarithmic subband decomposition (notated by 3D-DFT-C-LSD), and the proposed local 3D-DFT spectrum (notated by 3D-DFT-LS) as well as local 3D-DFT cepstrum (notated by 3D-DFT-LC). We can see that, the proposed local 3D-DFT descriptor outperforms their corresponding 3D-DFT descriptors.

Table 2 compares the proposed local 3D-DFT descriptors with/without performing IDT operation before feature extraction. It can be seen that by performing IDT operation to get a smoothed voxel representation of each 3D model, we can remove those high-frequency artifacts and thus will achieve higher retrieval accuracy.

Table 1. Comparison of different 3D-DFT descriptors on the PSB database in terms of the $D C G$ score. The parentheses show the setting of the parameters.

\begin{tabular}{ll}
\hline \multicolumn{1}{c}{ Descriptor } & DCG \\
\hline 3D-DFT-S $(M=16)$ & 64.63 \\
3D-DFT-S-USD $(W=H=L=32)$ & 65.54 \\
3D-DFT-S-LSD $(W=H=L=12)$ & 65.96 \\
3D-DFT-C $(D=16)$ & 63.14 \\
3D-DFT-C-USD $(W=H=L=32, D=16)$ & 64.67 \\
3D-DFT-C-LSD $(W=H=L=12, D=8)$ & 62.80 \\
3D-DFT-LS $(M=16)$ & $\mathbf{6 7 . 4 7}$ \\
3D-DFT-LC $(D=8)$ & $\mathbf{6 7 . 2 6}$ \\
\hline
\end{tabular}

Table 2. Comparison of the proposed local 3D-DFT descriptors with/without performing IDT operation. The parentheses show the setting of the parameters.

\begin{tabular}{ll}
\hline \multicolumn{1}{c}{ Descriptor } & DCG \\
\hline 3D-DFT-LS $(M=16)$ & 67.47 \\
3D-DFT-LC $(D=8)$ & 67.26 \\
3D-DFT-LS + IDT $(M=16)$ & $\mathbf{6 9 . 2 4}$ \\
3D-DFT-LC + IDT $(D=16)$ & $\mathbf{6 8 . 2 9}$ \\
\hline
\end{tabular}




\section{Conclusions}

In this paper, local 3D-DFT spectral descriptor and local 3D-DFT cepstral descriptor are proposed for 3D model retrieval. First, the set of voxels of a 3D model in the $3 \mathrm{D}$ space is divided into 8 octants. 3D-DFT spectral features and 3D-DFT cepstral features are extracted from each octant and combined to form the local 3D-DFT spectral descriptor and local 3D-DFT cepstral descriptor. Experiments conducted on the Princeton Shape Benchmark (PSB) database have shown that our proposed local 3D-DFT descriptors outperform other 3D-DFT descriptors.

\section{Acknowledgment}

This research was supported in part by the Ministry of Science and Technology of R.O.C. under contract MOST 103-2221-E-216-014.

\section{References}

(1) T. Funkhouser, P. Min, M. Kazhdan, J. Chen, A. Halderman, D. Dobkin, and D. Jacobs, "A search engine for 3D models", ACM Trans. on Graphics, Vol. 22, pp. 83-105, 2003.

(2) N. Iyer, S. Jayanti, K. Lou, Y. Kalyanaraman, and K. Ramani, "Three-dimensional shape searching: state-of-the-art review and future trends", Computer-Aided Design, Vol. 37, No. 5, pp. 509-530, 2005.

(3) Y. C. Pu, W. C. Du, C. H. Huang and C. K. Lai, "Invariant feature extraction for 3D model retrieval: an adaptive approach using Euclidean and topological metrics", Computers and Mathematics with Applications, Vol. 64, pp. $1217-1225,2012$

(4) S. Kawamura, K. Usui, T. Furuya and R. Ohbuchi, "Local geometrical feature with spatial context for shape-base 3D model retrieval", in Proc. 5th Eurographics conf. on $3 D$ Object Retrieval, pp. 55-58, 2012.

(5) Y. Ohishi, T. Furuya and R. Ohbuchi, "Non-rigid 3D model retrieval using set of local statistical feature", in Proc. IEEE Int. Conf. on Multimedia and Expo (ICME), Sep 26, 2012.

(6) X. Pan, Q. You, Z. Liu and Q. H. Chen, "3D shape retrieval by Poisson histogram", Pattern Recognition Letters, Vol. 32, Issue 6, pp. 787-794, 2011.

(7) Y. S. Park, Y. I. Yun and J. S. Choi, "A new shape descriptor using sliced image histogram for 3D model retrieval", IEEE Trans. on Consumer Electronics, Vol. 55, Issue 1, pp. 240-247, 2009

(8) M. Reisert and H. Burkhardt, "Second order 3D shape features: an exhaustive study", Computers \& Graphics, Vol. 30, No 2, pp. 197-206, 2006

(9) J. L. Shih, C. H. Lee and J. T. Wang, "3D Object Retrieval System Based on Grid D2", Electronics Letters, Vol. 41, No. 4, pp. 23-24, 2005.

(10) C. Y. Ip, L. Sieger, W. C. Regli and A. Shokoufandeh, "Automated learning of model classifications," in Proc. Solid Modeling, pp. 322-327, 2003.

(11) C. Y. Ip, D. Lapadat, L. Sieger and W. C. Regli, "Using shape distributions to compare solid models", in Proc. Solid Modeling, pp. 273-280, 2002.
(12) E. Wahl, G. Hillenbrand and G. Hirzinger, "Surflet-pair-relation histograms- a statistical 3D-shape representation for rapid classification", in Proc. 3D Digital Imaging and Modeling, pp. 474-481, 2003.

(13) R. Osada, T. Funkhouser, B. Chazelle and D. Dobkin, "Shape distributions", ACM Trans. on Graphics, Vol. 21, No. 4, pp. 807-832, 2002.

(14) B. S. Manjunath, P. Salembier and T. Sikora, "Introduction to MPEG-7 multimedia content description interface", John Wiley \& Sons, 2002.

(15) MPEG Video Group, "MPEG-7 Visual part of eXperimetation Model Version 9.0", ISO/IEC JTC1/SC29/WG11/N3914, Jan. 2001.

(16) T. Zaharia and F. J. Preteux, "Shape-based retrieval of 3D mesh models", in Proc. IEEE Int. Conf. Multimedia and Expo, Vol. 1, pp. 437-440, 2002.

(17) C. Zhang and T. Chen, "Efficient feature extraction for 2D/3D objects in mesh representation," in Proc. IEEE Int. Conf. Image Processing (ICIP), Thessaloniki, Greece, pp. 935-938, 2001.

(18) D. V. Vranic and D. Saupe, "3D model retrieval," in Proc. Spring Conf. Computer Graphics and its Applications, Budmerice Manor, Slovakia, pp. 89-93, May. 2000.

(19) M. Ankerst, G. Kastenmuller, H. P. Kriegel and T. Seidl, “3D shape histograms for similarity search and classification in spatial databases", in Proc. Symposium on Large Spatial Databases, pp. 207-226, 1999.

(20) H. Dutagaci, B. Sankur and Y. Yemez, "Subspace methods for retrieval of general 3D models", Computer Vision and Image Understanding, Vol. 114, No. 8, pp. 865-886, 2010.

(21) C. B. Akgul, B. Sankur, Y. Yemez and F. Schmitt, "3D model retrieval using probability density-based shape descriptors", IEEE Trans. Pattern Analysis And Machine Intelligence, Vol. 31, No. 6, pp. 1117-1133, June 2009.

(22) P. Papadakis, I. Pratikakis, S. Perantonis and T. Theoharis, "Efficient 3D shape matching and retrieval using a concrete radialized spherical projection representation", Pattern Recognition, Vol. 40, pp. 2437-2452, 2007.

(23) H. Laga, H. Takahashi and M. Nakajima, "Spherical wavelet descriptors for content-based 3D model retrieval", in Proc. IEEE Int. Conf. Shape Modeling and Applications, 2006.

(24) L. Ying, L. Demanet and E. Candes, "3D discrete curvelet transform”, Wavelets XI, Vol. 5914, No. 1, pp. 351-361, Sep 2005.

(25) M. Kazhdan, T. Funkhouser and S. Rusinkiewicz, "Rotation invariant spherical harmonic representation of 3D shape descriptors", in Proc. Symposium Geometry Processing, 2003.

(26) D. V. Vranic, D. Saupe and J. Richter, "Tools for 3D-object retrieval : Karhunen-Loeve transform and spherical harmonics", in Proc. IEEE Workshop Multimedia Signal Processing, pp. 293-298, 2001.

(27) D. V. Vranic and D. Saupe, "3D shape descriptor based on 3D Fourier transform", in Proc. EURASIP Conf. Digital Signal Processing for Multimedia Communications and Services, Budapest, Hungary, pp. 271-274, Sep. 2001.

(28) J. Ricard, D. Coeurjolly and A. Baskurt, "ART extension for description, indexing and retrieval of 3D objects", Pattern Recognition, Vol. 3, pp.79-82, 2004.

(29) Y. Gao, J. Tang, R. Hong, S. Yan, Q. Dai, N. Zhang and T. S. Chua, "Camera constraint-free view-based 3-D object retrieval", IEEE Trans. on Image Processing, Vol. 21, No. 4, pp. 2269-2281, 2012.

(30) Y. Gao, M. Wang, D. Tao, R. Ji and Q. Dai, "3D object retrieval and recognition with hypergraph analysis", IEEE Trans. on Image Processing, Vol. 21 , No. 9, pp. 4290-4303, Sep. 2012. 
(31) Q. Xiao, H. Wang, F. Li and Y. Gao, "3D object retrieval based on a graph model descriptor", Neurocomputing, Vol. 74, No. 17, pp. 3486-3493, 2011.

(32) Y. Gao, Q. H Dai, M. Wang and N. Zhang, "3D model retrieval using weighted bipartite graph matching", Signal Processing: Image Communication, Vol. 26, No. 1, pp. 39-47, 2011.

(33) J. L. Shih, C. H. Lee and C. H. Chuang, "A 3D model retrieval approach based on the combination of PCA plane projections", Journal of Information Technology and Applications, Vol. 5, No. 2, pp. 46-102, 2011.

(34) Q. Zhang, J. Jia and H. Li, “A GPU based 3D object retrieval approach using spatial shape information", in Proc. IEEE Int. Symposium Multimedia, pp. 212-219, 2010.

(35) Z. Lian, A. Godil and X. Sun, "Visual similarity based 3D shape retrieval using bag-of-features", in Proc. Int. Shape Modeling Conf., 2010.

(36) Y. Gao, Q. H. Dai and N. Y. Zhang, "3D model comparison using spatial structure circular descriptor", Pattern Recognition, Vol. 43, pp. 1142-1151, 2010.

(37) P. Papadakis, I. Pratikakis, T. Theoharis and S. Perantonis, "PANORAMA: a 3D shape descriptor based on panoramic views for unsupervised 3D object retrieval", Int. Journal of Computer Vision, Vol. 89, pp. 177-192, 2010.

(38) A. Mademlis, P. Daras, D. Tzovaras and M. G. Strintzis, "3D object retrieval using the 3D shape impact descriptor", Pattern Recognition, Vol. 42, pp. 2447-2459, 2009.

(39) J. L. Shih and H. Y. Chen, "A 3D model retrieval approach using the interior and exterior 3D shape information", Multimedia Tools and Applications, Vol. 43, pp. 45-62, 2008.

(40) A. Tatsuma and M. Aono, "Multi-Fourier spectra descriptor and augmentation with spectral clustering for 3D shape retrieval”, The Visual Computer, Vol. 25, No. 8, pp. 785-804, June 2009.

(41) C. T. Kuo and S. C. Cheng, "3D model retrieval using principal plane analysis and dynamic programming", Pattern Recognition, Vol. 40, No. 2, pp. 742-755, 2007.

(42) J. L. Shih and W. C. Wang, "A 3D model retrieval approach based on the principal plane descriptor", in Proc. Int. Conf. Innovative Computing, Information and Control, 2007.

(43) D. Zarpalas, P. Daras, A. Axenopoulos, D. Tzovaras and M. G. Strintzis, "3D model search and retrieval using the spherical trace transform", EURASIP Journal on Applied Signal Processing, Vol. 2007, pp. 207-207, 2007.

(44) M. Chaouch and A. Verroust-Blondet, "A new descriptor for 2D depth image indexing and 3D model retrieval", in Proc. IEEE Int. Conf. on Image Processing, Vol. 6, pp. 373-376, 2007.

(45) J. L. Shih, C. H. Lee and J. T. Wang, "A new 3D Model retrieval approach based on the elevation descriptor", Pattern Recognition, Vol. 40, No. 1, pp. 283-295, 2007.

(46) J. Assfalg, A. D. Bimbo and P. Pala, "Content-based retrieval of 3D models through curvature maps: A CBR approach exploiting media conversion," Multimedia Tools and Applications, Vol. 31, No. 1, pp. 29-50, 2006.

(47) J. Assfalg, A. D. Bimbo and P. Pala, "Curvature maps for 3D CBR”, in Pro. Multimedia and Expo, Vol. 2, pp. 301-304, 2003.

(48) J. Assfalg, A. D. Bimbo and P. Pala, "Retrieval of 3D objects using curvature maps and weighted walkthroughs", in Proc. Image Analysis and Processing, pp. 348-353, 2003.

(49) J. L. Shih and H. Y. Chen, "3D model retrieval based on grid sphere and dodecahedral silhouette descriptors", in Proc. 9th Joint Conf. on Information Science, Taiwan, 2006.

(50) D. V. Vranic, "3D model retrieval," Ph. D. Thesis, University of Leipzig, 2004.
(51) D. Y. Chen, X. P. Tian, Y. T. Shen and M. Ouhyoung, "On visual similarity based 3D model retrieval", Computer Graphics Forum, Vol. 22, No. 3, pp. 223-232, 2003.

(52) B. J. Super and H. Lu, "Evaluation of a hypothesizer for silhouette-based 3-D object recognition", Pattern Recognition, Vol. 36, pp. 69-78, 2003.

(53) Y. Liu, X. D. Zhang, Z. M. Li and H. Li, "Extended cone-curvature based salient points detection and 3D model retrieval", Multimedia Tools and Applications, Vol. 64, pp. 671-693, 2013.

(54) M. C. Changand B. B. Kimia, "Measuring 3D shape similarity by graph-based matching of the medial scaffolds", Computer Vision and Image Understanding, Vol. 115, No. 5, pp. 707-720, 2011.

(55) T. Tung and F. Schmitt, "The augmented multiresolution Reeb graph approach for content-based retrieval of 3D shapes", Int. Journal of Shape Modeling, Vol. 11, No. 1, 2005

(56) H. Sundar, D. Silver, N. Gagvani and S. Dickinson, "Skeleton based shape matching and retrieval", in Proc. Int. Conf. Shape Modeling, pp. 130-139, 2003.

(57) J. L. Shih, C. H. Lee, C. W. Wang and Y. H. Liu, “A 3D model retrieval approach using 3D-DFT spectrum and cepstrum", in Proc. Conf. Computer Vision, Graphics, and Image Processing, Taiwan, 2014.

(58) P. Shilane, P. Min, M. Kazhdan, and T. Funkhouser, "The Princeton shape benchmark", in Proc. Shape Modeling, pp. 167-178, 2004.

(59) C. B. Akgul, B. Sankur, Y. Yemez, and F. Schmitt, "3D model retrieval using probability density-based shape descriptors," IEEE Trans. on Pattern Analysis and Machine Intelligence, Vol. 31, No. 6, pp. 1117-1133, June, 2009. 